\title{
Overview of Structural Reliability Analysis Methods - Part III: Global Reliability Methods
}

\author{
ChangWu HUANG ${ }^{1,}{ }^{*}$, Abdelkhalak El Hami ${ }^{1}$ and Bouchaïb Radi ${ }^{2}$ \\ ${ }^{1}$ Normandie Université, INSA Rouen, LOFIMS, 76000 Rouen, France. \\ *Corresponding author: ChangWu.Huang.PRC@gmail.com \\ ${ }^{2}$ LIMII, FST Settat, BP: 577, Route de Casa, Settat, Morocco.
}

\begin{abstract}
In Part III of the overview of structural reliability analysis methods, global reliability methods, which are based on global approximation model of performance function using Gaussian process model, are reviewed. Gaussian process model is the basis for these global reliability methods. This category of methods, firstly, approximates the performance function by Gaussian process model, and then perform sampling methods based on the built surrogate model to calculate the failure probability. The computational cost is significantly reduced with the aid of surrogate model, since the surrogate model is cheap to evaluate. Additionally, global reliability methods can give accurate results because Gaussian process model can adequately model the nonlinear limit state function. After the introduction of Gaussian process model, two global reliability methods, EGRA and AK-MCS are described and illustrated by an example.

KEYWORDS. Reliability Analysis, Gaussian Process Model, Global Reliability Methods, Monte Carlo Simulation.
\end{abstract}

\section{Introduction}

Local reliability methods are computationally efficient. However, these methods may fail in situations such as the limit state function is non-smooth or the limit state is multimodal (multiple MPPs). Furthermore, even local reliability methods can work, their results are not generally accurate enough due to their inadequate approximation of the limit state function, especially when the limit state if highly nonlinear. Finally, in engineering applications, the performance functions are often characterized by implicit response functions that are expensive to evaluate and perhaps of nonlinearity. In these conditions, MVFOSM, FORM and SORM of local reliability methods are difficult to be performed. Although the RSM can work, but it may not give promising results.

We are then forced to revert to simulation methods (or called sampling methods), which is not limited by distributions of random variable, the shape of limit state function or the MPP convergence. The main problem of sampling methods is that they typically require a large number of response function evaluations, which makes them impractical if the response function is expensive to evaluate. Therefore, a reliability analysis method that is both efficient when applied to expensive performance functions and accurate for a response function of any arbitrary shape is needed.

Recently, many researchers devote to this kind of reliability methods, which is called global reliability methods. Two typical and mature approaches of this kind of method are EGRA (efficient global reliability analysis) [1] and AK-MCS (Active learning reliability method combining Kriging and Monte Carlo simulation) [2]. In these two approaches, the Kriging or Gaussian Process model is used as a surrogate model to substitute for the expensive response function. An iterative process is firstly used to construct the global surrogate that is accurately depicted the limit state. Then this surrogate model is used to calculate the failure probability by any sampling method. The main difference between EGRA and AK-MCS is the learning function used in the algorithm, which determine the best next point to evaluate on the performance function and add to the set of training data.

In the following of this paper, the Gaussian process model (or Kriging model) is firstly presented in Section 2. Then the two approaches, EGRA and AK-MCS, are separately detailed in Section 30 and Section 40. After an example in Section 50, a summary is given in Section 60. 


\section{Gaussian Process Models}

A Gaussian Process (GP) is a collection of random variables, any finite number of which have (consistent) joint Gaussian distribution [3]. A Gaussian process is completely specified by its mean function and covariance function. The advantages of GP models are that they not only provide the best estimate value at an untired point, but also give an estimate of the prediction variance. This variance presents the prediction uncertainty, which depends on the number and location of training data points. Here, Gaussian process is used to model the performance function in a reliability problem. The basic idea is that the performance function values $G(\mathbf{X})$ at different values of input random variables $\mathbf{X}$ can be modeled as a Gaussian random field. Specifically, in EGRA and AK-MCS, the performance function $G(\mathbf{X})$ is modeled by:

$$
G(\mathbf{x})=\mathbf{h}(\mathbf{x})^{\mathrm{T}} \beta+Z(\mathbf{x})
$$

where $\mathbf{h}(\mathbf{x})$ are a set of fix basis functions, $\beta$ is a vector of regression coefficients, $\mathbf{h}(\mathbf{x})^{\mathrm{T}} \beta$ is the trend of the model, and $Z(\mathbf{x})$ is a zero mean GP. The trend of the model can be assumed to be any function, but it can also be taken as a constant value [3]. In these two approaches, the trend is assumed constant and $\beta$ is calculated by Generalized Least Squares estimate [4]. The covariance between two points $\mathbf{x}^{(p)}$ and $\mathbf{x}^{(q)}$ of Gaussian process $Z(\mathbf{x})$ is defined by

$$
\operatorname{Cov}\left[Z\left(\mathbf{x}^{(p)}\right), Z\left(\mathbf{x}^{(q)}\right)\right]=\sigma_{Z}^{2} R\left(\mathbf{x}^{(p)}, \mathbf{x}^{(q)}\right)
$$

where $\sigma_{z}^{2}$ is the process variance and $R(\cdot)$ is the correlation function. The used squared-exponential correlation function, which is commonly wide-used one, is formulated by

$$
R\left(\mathbf{x}^{(p)}, \mathbf{x}^{(q)}\right)=\prod_{i=1}^{n} \exp \left[-\theta_{i}\left(x_{i}^{(p)}-x_{i}^{(q)}\right)^{2}\right]
$$

where $n$ is the dimensionality of the random variables, $x_{i}^{(p)}$ and $x_{i}^{(q)}$ are the $i$-th coordinates of the point $\mathbf{x}^{(p)}$ and $\mathbf{x}^{(q)}$, and $\theta_{i}$ is a scale parameter that indicates the correlation between the points within $i$-th dimension. The scale parameters $\theta_{i}$ are collected in vector $\theta=\left[\theta_{1}, \cdots, \theta_{n}\right]$ hereafter.

The goal of Gaussian process modeling is to construct a GP model for prediction based on a given set of training points. Suppose we have the training data set $\mathcal{D}=\left\{\left(\mathbf{x}^{(i)}, y^{(i)}\right) \mid i=1, \cdots, k\right\}$, where $\mathbf{x}^{(i)}$ denote the input points from a design of experiments and $y^{(i)}=g\left(\mathbf{x}^{(i)}\right)$ are their corresponding performance function values; the column vector inputs for $k$ sampled points are aggregated in the $n \times k$ design matrix $\mathbf{X}_{\mathrm{s}}$ and the corresponding performance function values are collected in the vector $\mathbf{Y}_{\mathrm{S}}=\left[y^{(1)}, \cdots, y^{(k)}\right]$. With the training data, the estimates of $\beta$ and $\sigma_{z}^{2}$ are given by:

$$
\begin{aligned}
& \hat{\boldsymbol{\beta}}=\left(\mathbf{F}^{\mathrm{T}} \mathbf{R}^{-1} \mathbf{F}\right)^{-1} \mathbf{F}^{\mathrm{T}} \mathbf{R}^{-1} \mathbf{Y}_{\mathrm{S}} \\
& \hat{\sigma}_{Z}^{2}=\frac{1}{k}\left(\mathbf{Y}_{\mathrm{S}}-\mathbf{F}^{\mathrm{T}} \boldsymbol{\beta}\right)^{\mathrm{T}} \mathbf{R}^{-1}\left(\mathbf{Y}_{\mathrm{S}}-\mathbf{F}^{\mathrm{T}} \boldsymbol{\beta}\right)
\end{aligned}
$$

where $\mathbf{F}$ is an $k \times q$ matrix with rows $\mathbf{h}\left(\mathbf{x}^{(i)}\right)^{\mathrm{T}}$ (the basis function for training point $i$ containing $q$ terms; for a constant trend $q=1$ ), $\mathbf{R}$ is a $k \times k$ matrix of correlation between each pair of points in the training data set. When the trend function is set as constant, we have $\mathbf{F}=\mathbf{1}$, here $\mathbf{1}$ is the column vector filled with 1 of length $k$. 
Since $\hat{\beta}$ and $\hat{\sigma}_{z}^{2}$ in Equations (4) and (5) depend on the correlation parameters $\theta$ through the correlation matrix R, parameters $\theta$ need to be estimated firstly. The correlation parameters $\theta$ can be determined by maximum likelihood estimation [5]:

$$
\left\{\begin{array}{l}
\hat{\boldsymbol{\theta}}=\underset{\boldsymbol{\theta}}{\arg \max } L(\boldsymbol{\theta})=-\frac{n}{2} \ln (2 \pi)-\frac{n}{2} \ln \left(\hat{\sigma}_{Z}^{2}\right)-\frac{1}{2} \ln |\mathbf{R}| \\
\text { s.t. } \theta_{l}>0, l=1,2, \cdots, n
\end{array}\right.
$$

where $L(\theta)$ is the ln-likelihood function of $\theta$. With the estimation $\hat{\theta}, \hat{\beta}$ and $\hat{\sigma}_{Z}^{2}$ can be determined through Equations (4) and (5). Having all the parameters $\hat{\theta}, \hat{\beta}$ and $\hat{\sigma}_{z}^{2}$ in the model, now, the prediction $\hat{G}\left(\mathbf{x}^{*}\right)$ of the response value at untired point $\mathbf{x}^{*}$ can be given by its mean value $\mu_{G}\left(\mathbf{x}^{*}\right)$, also called the Best Linear Unbiased Predictor (BLUP), and variance $\sigma_{G}^{2}\left(\mathbf{x}^{*}\right)$ as:

$$
\begin{aligned}
& \mu_{G}\left(\mathbf{x}^{*}\right)=\mathbf{h}\left(\mathbf{x}^{*}\right)^{\mathrm{T}} \hat{\boldsymbol{\beta}}+\mathbf{r}\left(\mathbf{x}^{*}\right)^{\mathrm{T}} \mathbf{R}^{-1}\left(\mathbf{Y}_{\mathrm{S}}-\mathbf{F}^{\mathrm{T}} \hat{\boldsymbol{\beta}}\right) \\
& \sigma_{G}^{2}\left(\mathbf{x}^{*}\right)=\hat{\sigma}_{Z}^{2}-\left[\begin{array}{ll}
\mathbf{h}\left(\mathbf{x}^{*}\right)^{\mathrm{T}} & \mathbf{r}\left(\mathbf{x}^{*}\right)^{\mathrm{T}}
\end{array}\right]\left[\begin{array}{ll}
\mathbf{0} & \mathbf{F}^{\mathrm{T}} \\
\mathbf{F} & \mathbf{R}
\end{array}\right]^{-1}\left[\begin{array}{l}
\mathbf{h}\left(\mathbf{x}^{*}\right) \\
\mathbf{r}\left(\mathbf{x}^{*}\right)
\end{array}\right]
\end{aligned}
$$

where $\mu_{G}\left(\mathbf{x}^{*}\right)$ and $\sigma_{G}^{2}\left(\mathbf{x}^{*}\right)$ are the mean value and variance of the predictor $\hat{G}\left(\mathbf{x}^{*}\right), \mathbf{r}\left(\mathbf{x}^{*}\right)$ is the vector containing the correlation between $\mathbf{x}^{*}$ and each $\mathbf{x}^{(i)}$ of the $k$ training points.

Gaussian process regression model is an exact interpolation method. This means that the error in the model at known points is zero. Therefore, the variance in the Gaussian distribution at the training point is zero. This enables to quantify the uncertainty of local predictions by their variances. These properties provide good index to improve a design of experiments and update the surrogate model. Of course, EGRA and AK-MCS indeed take advantages of these properties.

\section{Efficient Global Reliability Analysis (EGRA)}

In efficient global reliability analysis (EGRA) proposed in reference [6], a so-called expected feasibility function (EFF) is used to select the location at which a new training point should be added. The EFF indicates how well the true value of the response is expected to satisfy the equality constrain $G(\mathbf{x})=z$. Thus, the EFF is formed by integrating over a region in the vicinity of the threshold values $z \pm \varepsilon$ :

$$
\operatorname{EFF}(\mathbf{x})=\int_{z-\varepsilon}^{z+\varepsilon}[\varepsilon-|z-\hat{G}(\mathbf{x})|] f_{G}(\hat{G}(\mathbf{x})) \mathrm{d} \hat{G}(\mathbf{x})
$$

where $\hat{G}(\mathbf{x})$ is the GP prediction, which follows a Gaussian distribution with mean $\mu_{G}(\mathbf{x})$ and variance $\sigma_{G}^{2}(\mathbf{x})$ defined by Equation (7) and (8), $f_{G}(\hat{G}(\mathbf{x}))$ is the density distribution function of $\hat{G}(\mathbf{x})$. We can see that a point with higher EFF value its predictor is closer to the equality constrain. Therefore, the point which has maximum EFF value is find and added to the design of experiments. Considering $\hat{G}(\mathbf{x}) \sim \mathcal{N}\left[\mu_{G}(\mathbf{x}), \sigma_{G}^{2}(\mathbf{x})\right]$, the above defined expected feasibility function (EFF) can be analytically expressed as:

$$
\begin{aligned}
\operatorname{EFF}(\mathbf{x})= & {\left[\mu_{G}(\mathbf{x})-z\right]\left[2 \Phi\left(\frac{z-\mu_{G}(\mathbf{x})}{\sigma_{G}(\mathbf{x})}\right)-\Phi\left(\frac{(z-\varepsilon)-\mu_{G}(\mathbf{x})}{\sigma_{G}(\mathbf{x})}\right)-\Phi\left(\frac{(z+\varepsilon)-\mu_{G}(\mathbf{x})}{\sigma_{G}(\mathbf{x})}\right)\right] } \\
& -\sigma_{G}(\mathbf{x})\left[2 \varphi\left(\frac{z-\mu_{G}(\mathbf{x})}{\sigma_{G}(\mathbf{x})}\right)-\varphi\left(\frac{(z-\varepsilon)-\mu_{G}(\mathbf{x})}{\sigma_{G}(\mathbf{x})}\right)-\varphi\left(\frac{(z+\varepsilon)-\mu_{G}(\mathbf{x})}{\sigma_{G}(\mathbf{x})}\right)\right] \\
& +\varepsilon\left[\Phi\left(\frac{(z+\varepsilon)-\mu_{G}(\mathbf{x})}{\sigma_{G}(\mathbf{x})}\right)-\Phi\left(\frac{(z-\varepsilon)-\mu_{G}(\mathbf{x})}{\sigma_{G}(\mathbf{x})}\right)\right]
\end{aligned}
$$


where $\varphi(\cdot)$ and $\Phi(\cdot)$ are the PDF and CDF of Standard Normal distribution, $\varepsilon$ is proportional to the standard deviation of the GP predictor, i.e., $\varepsilon \propto \sigma_{G}(\mathbf{x})$.In case of reliability problem, the threshold $z=0$, and the EFF is built with $\varepsilon=2 \sigma_{G}(\mathbf{x})$.

The EFF provides a balance between exploiting areas of the design space where good solutions have been found, and exploring areas of the design space where the uncertainty is high. Points where the expected value is close to the threshold $\left(\mu_{G}(\mathbf{x}) \approx z\right)$ and points with a large uncertainty in the prediction will have large expected feasibility values [7]. The algorithm of EGRA is formed as [8]:

1. Generate a small number of random samples from the true response function.

a) Only $(n+1)(n+2) / 2$ samples are used (where $\mathrm{n}$ is the number of random variables). This initial selection is arbitrary, but the number of samples required to define a quadratic polynomial is used as a convenient rule of thumb.

b) The samples uniformly span the random variable space over the bounds $\pm 5 \sigma$, though the bounds of this search space can be adjusted if needed.

c) Latin hypercube sampling (LHS) is used to generate the samples.

2. Construct an initial Gaussian process model from these samples.

3. Find the point with maximum expected feasibility.

a) The expected feasibility function is built with $\varepsilon=2 \sigma_{G}(\mathbf{x})$.

b) To locate the global optimum of this multimodal function is, the DIRECT[9] method is used.

c) If the maximum expected feasibility is less than 0.001 , the model has converged. Go to step 6 .

4. Evaluate the true response function at this point.

5. Add this new sample to the previous set of training data and build a new GP model. Go to step 3 .

6. This surrogate model is then used to calculate the probability of failure using any sampling method.

Typically, the multimodal adaptive importance sampling (MAIS) is used reduce the sampling cost. Other sampling methods, such as Latin Hypercube Sampling (LHS), of course, can be applied.

\section{Active Learning Reliability Method combining Kriging and Monte Carlo Simulation (AK-MCS)}

The AK-MCS (Active Learning Reliability Method combining Kriging and Monte Carlo Simulation), proposed by B. Echard, N. Gayton and M. Lemaire in reference [2], is an iterative approach based on Monte Carlo Simulation and Kriging metamodel to assess the reliability of structures in a more efficient way. AK-MCS and EGRA use the Kriging (or Gaussian Process) model in the same way to avoid expensive evaluations of responses on true performance function. The key difference between these two approaches lies in that EGRA approximates the limit state in the whole space while AK-MCS focus on the accuracy on sign of the performance function values among a Monte Carlo population. This difference is exhibited through the difference in learning functions. In EGRA the expected feasibility function (EFF) defined in Equation (9) is used, but in AK-MCS a newly defined learning function $U(\mathbf{x})$ is applied.

The underling idea of the proposed learning function $U$ is driven by that in Monte Carlo simulation only the sign of the performance function is important, i.e., only the sign of the response values contribute to the failure probability calculated by MCS. In order to grantee accuracy on the sign, the points with a high potential risk of crossing the limit state surface $g(\mathbf{x})=0$ need to be added to the design of experiments, because the uncertainty on these points can cause their predictors change from positive to negative (or from negative to positive), which then leads to a modification of the failure probability. 
It can be concluded that points with characteristics, specifically, be close to the limit state, have important uncertainty (great Kriging variance) or both at the same, show high potential risk of making a mistake on the sign of the predictor. Consequently, the learning function $U(\mathbf{x})$ is postposed as:

$$
\left|\mu_{G}(\mathbf{x})\right|-U(\mathbf{x}) \sigma_{G}(\mathbf{x})=0
$$

where $\mu_{G}(\mathbf{x})$ and $\sigma_{G}(\mathbf{x})$ are the predictor mean value and standard deviation defined by Equation (7) and (8).

Form its definition, a point with lower $U(\mathbf{x})$ value means it has high risk of making a mistake on the sign. So, in each iteration, the point that has minimum learning function $U(\mathbf{x})$ in Monte Carlo population is selected to add into the design of experiments. The stopping criterion for this learning iteration is $\min (U(\mathbf{x})) \geq 2$, which means that if after an iteration that the minimum learning function value is not less than 2, we stop the learning process. A detailed version of the algorithm given in [2] is summarized here:

1. Generation of a Monte Carlo population in the design space. It is named $\mathbf{S}$ and of the size $N_{\mathrm{MC}}$, i.e., $\mathbf{S}=\left\{\mathbf{x}^{(1)}, \cdots, \mathbf{x}^{\left(N_{\mathrm{MC}}\right)}\right\}$. This population remains the same during the whole process of leaning in AK-MCS unless Step 9 is reached.

2. Definition of the initial design of experiment (DOE). The initial DOE is formed by randomly selecting $N_{1}\left(N_{1} \ll N_{\text {MC }}\right)$ points among the Monte Carlo population $\mathbf{s}$. Then, these points in DOE are evaluated by the performance function, which have form the training data $\operatorname{set} \mathcal{D}=\left\{\left(\mathbf{x}^{(1)}, G\left(\mathbf{x}^{(1)}\right)\right), \cdots,\left(\mathbf{x}^{\left(N_{1}\right)}, G\left(\mathbf{x}^{\left(N_{1}\right)}\right)\right)\right\}$.

3. Computation of the Kriging model according to the training data. This can be performed by using the toolbox DACE [10].

4. Estimation of the failure probability by Kriging prediction. First, Kriging predictions (BLUP) $\mu_{G}\left(\mathbf{x}^{(i)}\right)$ for $i=1, \cdots, N_{\mathrm{MC}}$ given by Equation (7) are obtained owing to DACE. Then, the failure probability is estimated with the signs of these predictions. The number of predictions with negative sign or null is recorded as $N_{\mathrm{f}}$. Finally, the estimation of failure probability is

$$
\hat{p}_{\mathrm{f}}=N_{\mathrm{f}} / N_{\mathrm{MC}}
$$

5. Identification of the best next point in $\mathbf{S}$ to evaluate on the performance function and added to the training data set. The learning function $U(\mathbf{x})$ defined by Equation (11) is computed for each point of $\mathbf{s}$. The point with the smallest learning function value is taken as the best next point $\mathbf{x}^{*}$, i.e., $\mathbf{x}^{*}=\underset{x \in \mathrm{S}}{\arg \min } U(\mathbf{x})$.

6. Check the stopping condition on learning. Once the best next point $\mathbf{x}^{*}$ is fond, its corresponding learning function value $U\left(\mathbf{x}^{*}\right)$ is compared to the stopping criterion (In reference [2], the stopping learning function value is 2). If $U\left(\mathbf{x}^{*}\right) \leq 2$, the best next point will be added to the DOE and its response is evaluated by performance function; otherwise, turn to Step 8 .

7. Update of training data set and the Kriging model. If the stopping criterion is not satisfied, the best next point $\mathbf{x}^{*}$ and its corresponding performance function value $G\left(\mathbf{x}^{*}\right)$ are added to the training data set $\mathcal{D}$. And then, the method goes back to Step 3 to construct a new Kriging model in the light of the new training data set.

8. Computation of the coefficient of variation of the failure probability. After the stopping condition in Step 6 is satisfied, the learning process is stopped and the metamodel is said to be accurate enough on the performance function values' signs of the Monte Carlo population $\mathbf{S}$. After the failure probability is estimated by the final Kriging model, its coefficient of variation is computed as Equation (13). If $\operatorname{cov}_{\hat{p}_{\mathrm{r}}}<5 \%$, the result is acceptable, i.e., the population $\mathbf{S}$ is sufficiently large to obtain a low coefficient 
of variation of Kriging estimation of the failure probability, and the AK-MCS iteration process is ended; otherwise go to Step 9.

$$
\operatorname{cov}_{\hat{p}_{\mathrm{f}}}=\sqrt{\frac{1-\hat{p}_{\mathrm{f}}}{\hat{p}_{\mathrm{f}} N_{\mathrm{MC}}}}
$$

9. Update of the population. If the coefficient of variation is too large, $\mathbf{S}$ is augmented by combining new points from another Monte Carlo population, and the procedure goes to Step 4.

In AK-MCS, from above algorithm, the signs of the performance function's values among the Monte Carlo sampling set [11] are sincerely considered, and the failure probability is computed at each iteration. Comparing with EGRA, the convergence of the failure probability in terms of the number of calls of the performance function can be easier to followed; moreover, the optimization algorithm, such as DIRECT or other derivate-free optimization algorithms, does not need in finding the best next point because the learning is not performed on the whole design space but only among the population. In both EGRA and AK-MCS, even if the number of predictions by Kriging model may be large, the computation time of the predictions can be neglected compared to the time required to evaluate the performance function (generally calculated by computer code, such as finite element analysis).

\section{Illustrative Example}

This example is taken from [12] [13]. The performance function is

$$
G(\mathbf{X})=X_{1}^{3}+X_{1}^{2} X_{2}+X_{2}^{3}-18
$$

where $X_{1}$ and $X_{2}$ are normally distributed random variables with mean values and standard deviation $\mu_{X_{1}}=10, \sigma_{X_{1}}=5$ and $\mu_{X_{1}}=9.9, \sigma_{X_{1}}=5$; the variables are uncorrelated.

This example is solved by LHS and the two global reliability methods, EGRA and AK-MCS. In order to establish an accurate estimate of the true solution, 20 independent LHS simulations with sample size of $1 \times 10^{6}$ were performed. The average failure probability from these 20 simulations is considered as the "true" solution for this example. Because EGRA and AK-MCS are stochastic, these two methods were also run 20 times and the average probabilities are reported. In EGRA, with the converged GP model, the failure probability is estimated by $1 \times 10^{6}$ Latin Hypercube samples. In AK-MCS, the Monte Carlo population also has a size of $1 \times 10^{6}$. To measure the accuracy of the methods, two errors which were used in Reference [8] are reported for EGRA and AK-MCS results: the error in the average probability and the average of the absolute errors from 20 independent simulations.

The results are presented in Table 1 . In this table, performance function evaluations indicates the average number of performance function evaluations needed in each methods, $\bar{p}_{\mathrm{f}}$ is the average of $\hat{p}_{\mathrm{f}}$ from 20 simulations, error in $\bar{p}_{\mathrm{f}}$ is the absolute error of each method with respect to the "true" solution, and average of the error from $\hat{p}_{\mathrm{f}}$ is the average of the absolute errors of $\hat{p}_{\mathrm{f}}$ in each method from 20 Simulations with respect to the "true" solution.

\begin{tabular}{|c|c|c|c|c|}
\hline Reliability Method & Performance Function Evaluations & $\bar{p}_{\mathrm{f}}$ & Error in $\bar{p}_{\mathrm{f}}$ & Average of the Error from $\hat{p}_{\mathrm{f}}$ \\
\hline LHS Sampling & $1.0 \mathrm{E}+06$ & 0.005716 & "truth" & $0.8443 \%$ \\
\hline EGRA & 17.45 & 0.005729 & $0.2336 \%$ & $1.3854 \%$ \\
\hline AK-MCS & 20.65 & 0.005713 & $0.0437 \%$ & $0.8964 \%$ \\
\hline
\end{tabular}

Table 1. Results of the cubic function problem

From Table 1, firstly, one can find that the global reliability methods, EGRA and AK-MCS, both give accurate solutions for the highly nonlinear problem of this example. In this specific example, the solution of AK-MCS is a little more accurate than that of EGRA, which can be drawn from the error 
measures. Moreover, the global reliability methods require a small number of performance function evaluations. This is very efficient for problems with performance functions that are expensive to evaluate. Specifically, only 17.45 calls of performance function are needed in average for 20 runs of EGRA, whereas 20.65 times of performance function evaluations are required in average for 20 simulations of AK-MCS. This example has shown the advantages of global reliability methods in accuracy and efficiency.

\section{Summary}

The two approaches, EGRA and AK-MCS, of global reliability methods are promising and compelling for practical engineering reliability problems, in which the performance functions are known implicit and expensive to evaluate. Based on the sampling methods, global reliability methods are accurate as the same advantage of sampling methods. In addition, with the use of Gaussian process model (or called Kriging model), this kind of methods also are efficient. This category of reliability methods can be applied in expensive situations of reliability problems. From the comparison between EGRA and AK-MCS, AK-MCS seems easier and more efficient to perform in practice, because that the optimization algorithm for next best point finding is not required in AK-MCS while this is necessary in EGRA and that the learning function in AK-MCS really focuses on the limit state and thus little number of true performance function evaluations is need. In conclusion, the global reliability methods combine the advantages of metamodel and Monte Carlo simulation such that they are very suitable for implicit performance functions, especially when the performance function is expensive to evaluate and nonlinear. Furthermore, above presented global reliability methods also point out a promising perspective for future reliability analysis: to accurately and efficiently approximate the limit state or performance function and to enhance the sampling efficiency.

A comprehensive summarization of structural reliability analysis methods, from traditional MVFORM, FORM, SORM, RSM and MCS to advanced EGRA and AK-MCS, have been given in our work. This works will give readers a general view on reliability analysis and provides some valuable recommendations for application of reliability methods in practical problems. The underlying idea and concepts for each reliability analysis methods have been illustrated. This is useful for readers to understand and apply these methods. Moreover, the advantages and drawbacks of each method are also stated, which can give some guidance for users when they are struggling in choosing an appropriate method to solve a specified problem. It is of significant importance to include the most advance reliability methods EGRA and AK-MCS. These two advance methods not only are useful for practical problems but also indicate some directions and concepts for future development of reliability analysis.

\section{References}

[1] Bichon B.J., Eldred M.S., Swiler L.P., Mahadevan S., \& McFarland J.M., Multimodal reliability assessment for complex engineering applications using efficient global optimization. AIAA Paper No. AIAA-2007-1946, 2007.

[2] Echard B., Gayton N., \& Lemaire M., AK-MCS: an active learning reliability method combining Kriging and Monte Carlo simulation. Structural Safety, vol. 33, no. 2, pp. 145-154, 2011.

[3] Williams C.K., \& Rasmussen C.E., Gaussian processes for machine learning. The MIT Press, vol. 2, no. 3, pp.4, 2006.

[4] Cressie N., Statistics for spatial data (revised edition) John Wiley. New York, 1993.

[5] Huang C., Radi B., \& El Hami A., Uncertainty analysis of deep drawing using surrogate model based probabilistic method. The International Journal of Advanced Manufacturing Technology, pp. 1-12, 2016.

[6] Bichon B.J., Eldred M.S., Swiler L.P., Mahadevan S., \& McFarland J.M., Efficient global reliability analysis for nonlinear implicit performance functions. AIAA journal, vol. 46, no. 10, pp. 2459-2468, 2008.

[7] Bichon B.J., Mahadevan S., \& Eldred M., Reliability-based design optimization using efficient global reliability analysis. In Proceedings of the 50th AIAA/ASME/ASCE/AHS/ASC structures, structural dynamics, and material conference, May 2009.

[8] Bichon B.J., Efficient surrogate modeling for reliability analysis and design (Doctoral dissertation, Vanderbilt University), 2010. 
[9] Björkman M., \& Holmström K., Global Optimization Using the DIRECT Algorithm in Matlab. In in Matlab. Advanced Modeling and Optimization 1 (2), pp. 17-37, 1999.

[10] Lophaven S.N., Nielsen H.B., \& Sondergaard J., DACE-a Matlab Kriging toolbox; version 2; informatics and mathematical modelling. Technical University of Denmark, Technical Report No. IMM-TR-2002-12, 2002.

[11] Fauriat W., \& Gayton N., AK-SYS: an adaptation of the AK-MCS method for system reliability. Reliability Engineering \& System Safety, vol. 123, pp. 137-144, 2014.

[12] Wang L., \& Grandhi R.V., Safety index calculation using intervening variables for structural reliability analysis. Computers \& structures, vol. 59, no. 6, pp. 1139-1148, 1996.

[13] Kaymaz I., Application of kriging method to structural reliability problems. Structural Safety, vol. 27, no. 2, pp. 133-151, 2005. 